\title{
Agroforestería y ética ambiental en la gerencia de sistemas de producción
}

\author{
Pérez, Juan José* \\ Huerta, Isneira**
}

\section{Resumen}

El objetivo de este trabajo es realizar un análisis documental sobre el tema de la adopción de sistemas agroforestales en unidades de producción, desde la perspectiva de la ética ambiental en el paradigma de la postmodernidad. En ésta se plantean elementos teóricos y conceptuales de la ética ambiental y la agroforestería. Asimismo, se discute la necesidad de adoptar los sistemas agroforestales como alternativa tecnológica para proteger el medio ambiente y recuperar el equilibrio ecológico en las áreas dedicadas a la actividad agrícola. Además, se presentan algunos de los beneficios ambientales y económicos generados por estos sistemas y la importancia de su adopción como un componente ético en la gerencia de sistemas de producción.

Palabras clave: Agroforestería, ética ambiental, gerencia, sistemas de producción.

\section{Agroforestry and Environment Ethics in the Management of Production Systems}

\section{Abstract}

The objective of this paper is to abalyze a documentary review of the topic of the adoption of agro-forestry systems in farms, from the perspective of environmental ethics in the postmodern period. The study introduces theoretical and conceptual element as to environmental ethics and agro-forestry systems. Also, the adoption of agro-forestry systems is proposed as an alternative in order to protect the

Recibido: 01-09-03 . Aceptado: 02-01-31

* Ingeniero Agrónomo, Magíster en Gerencia de Agrosistemas. Profesor Agregado de la Facultad de Agronomía de LUZ. Investigador Acreditado en el PPI. Participante en el Doctorado de Ciencias Económicas de LUZ. E-mail: jjperez@luz.ve.

** Psicólogo, Especialista en Metodología de la Investigación y Profesora Invitada en el Programa de Gerencia de Agrosistemas de la Facultad de Agronomía de LUZ. E-mail: isneirahuerta@hotmail.com. 
environment and recover ecological equilibrium in areas dedicated to the agricultural activity. This study shows some of the environmental and economic benefits generated by these systems and the importance of their adoption as an ethical component in the management of production systems.

Key words: Agroforestry, environment ethics, management, production systems.

\section{Introducción}

La conservación del medio ambiente es de vital importancia para la supervivencia de la vida tal y como se conoce actualmente. El hombre a través de su acción, ha afectado significativamente, el equilibrio ecológico, y por consiguiente, ha deteriorado drásticamente los recursos naturales, base de su sustentación.

La agricultura es sólo una de las prácticas humanas que ha desvirtuado la relación del hombre con el ambiente. La deforestación, contaminación de aguas y suelos, son ejemplos del daño medioambiental producido por éste. Bajo el paradigma postmoderno, entendido como una concepción de la realidad compleja, cambiante y turbulenta, actualmente, la Agroforestería constituye una alternativa viable desde el punto económico, ambiental y ético, que pudiese contribuir a mejorar sustancialmente, el medio ambiente.

Sin embargo, la gerencia de las organizaciones agrícolas debe aprender y aprehender esta realidad, para poder así, tomar la decisión de adoptar esta tecnología conservacionista y comprometida con las generaciones presentes y futuras.

Esta decisión de la gerencia de las organizaciones agrícolas no resulta fácil, pero es perentorio. En el siglo XXI, la gerencia en los sistemas de producción debe responder a los cambios de paradigmas, de un mundo moderno a uno postmoderno, y en especial de pasar a ser, tal como lo plantea Díaz (2000:50), "una gerencia centrada en el hombre, en el ser humano como protagonista del proceso".

Precisamente, la siguiente es una investigación documental sobre el tema de la adopción de sistemas agroforestales como un componente ético en la gerencia de los sistemas de producción. La misma plantea elementos teóricos y conceptuales de la ética ambiental y la agroforestería. De igual forma, se discuten los aspectos relacionados con la ética y el ambiente desde la perspectiva de la Agroforestería. Se plantea el Desarrollo Sostenible como modelo de ética ambiental y se analiza el hombre frente a la Agricultura, como un enriquecimiento propio a expensas del empobrecimiento del ambiente. Finalmente, se presentan algunas reflexiones sobre la ética dentro de la postmodernidad, con el propósito de evidenciar la necesidad de la adopción de sistemas agroforestales.

\section{La ética ambiental}

Cuando se habla de ética, se busca la rectitud de los actos humanos, es decir, que éstos actúen conforme a un orden preestablecido (Cañas- Quiroz, 1998). Para cumplir su cometido la ética intenta adherirse a "principios" que expliquen los actos humanos según un punto de partida, un fundamento o una causa. Este principio u origen de los actos que trata de determinar la ética es siempre "último y 
racional", al tratar de ir hasta las raíces más profundas para alcanzar una mejor interpretación de las causas que impulsan el comportamiento del hombre.

Cabe entonces preguntarse, desde la óptica ética, ¿qué fundamenta el carácter irracional del hombre frente a su ambiente?; ¿por qué el hombre se cuida en extremo a sí mismo y no lo hace con respecto al ambiente que lo rodea?. La ética ambiental busca dar explicación a estas interrogantes, y asume una postura con respecto a la orientación de los actos del hombre frente a su ambiente.

En este sentido, Vidal y Santidrian (1990:94), sostienen que la Ética Ambiental, tiende a ofrecer al hombre nuevas formas de relacionarse con el entorno, así, han surgido propuestas conservacionistas, las cuales bajo un referente ecológico, muestren al hombre que el binomio hombre - ambiente puede resultar una relación armoniosa, de beneficios bilaterales y de garantía de sostenibilidad, como ocurre en el caso de los sistemas agroforestales, los cuales constituyen sistemas de manejo de los recursos naturales dinámicos, con bases ecológicas, que por medio de la integración de árboles en tierras de fincas y tierras abiertas, diversifican y sustentan la producción de pequeños productores para aumentar los beneficios sociales, económicos y ambientales (Leakey, 1997:23).

En un contexto amplio, la ética ambiental va ha generar un cuerpo de preceptos que establecen el vínculo entre las ciencias naturales y las sociales. Según Ferrete (1996), ella es el factor común para unir dos ámbitos tan alejados en sus medios y propósitos.
Es así como la Ética Ambiental demanda la ecología de la producción económica o economía ambiental, lo cual no significa sustituir la racionalidad económica por otra ecológica. Implica más bien que los enfoques económicos y ecológicos deben apoyarse en un referente moral, porque la historia ha enseñado que no se puede erigir la eficiencia económica como único criterio válido. Se trata, pues, de recuperar en el medio ambiente la primacía de lo ético, sin desmedro de la producción de bienes de consumo.

El hombre, como individuo y comunidad, establece una relación vital con su propio ambiente: necesita de él para vivir e imprime sobre él la importancia de su actividad. Esta relación se vincula directamente con el desarrollo de las comunidades en cualquier región o país del mundo. Por tanto, todo proceso de desarrollo está condicionado, en distinta medida, por el ambiente natural al que pertenece o se vincula una sociedad (Degiovanni, 2001).

Atendiendo la relación hombre ambiente, la Ética Ambiental, concibe al hombre como un ser orientado a la racionalización de los recursos naturales que utiliza, como por ejemplo cuando integra todos los recursos disponibles en los sistemas agroforestales, asegura la continuidad de la especie humana sobre el planeta. Sin embargo, el comportamiento del hombre pareciera opuesto a los preceptos de la ética ambiental al emplear la mayor parte de su pensamiento a la explotación productiva.

Los grupos humanos han promovido la expansión del poder, la tecnología y la creciente explotación de los recursos naturales. Al respecto, plantea Etkin 
(1993:31), que la visión del hombre frente al ambiente es perversa, porque la calidad de vida y el ambiente humano tienen grandes posibilidades de degradarse, a menos que en el contexto funcionen controles que operen bajo el modelo de la retroalimentación negativa para evitar la errática oscilación del ecosistema, entre periodos de abundancia y otros de escasez.

Aunado a lo anterior, cuando se adoptan sistemas agroforestales 0 alguna práctica de la agroforestería, se busca la permanencia de los agrosistemas en el tiempo, evitando así la relación de perversidad entre el hombre y su ambiente.

\section{Los sistemas agroforestales}

La definición de "sistemas agroforestales" incluye diferentes aspectos relacionados, principalmente, con el uso de especies vegetales arbóreas de la familia de las leguminosas, combinadas con otras especies vegetales y animales. El concepto de agroforestería o sistemas agroforestales, ha evolucionado a través del tiempo. Las primeras definiciones, formuladas entre 1977 y 1979, son atribuidas a varios autores, entre los que se encuentran Bane, Bael, Cote, Combe y Budowski (Iglesias, 1999:287).

Nair, citado por Burley y Speedy (1998), define la agroforestería como sistemas y tecnologías de uso del suelo en los cuales las especies leñosas perennes se utilizan en el mismo sistema de manejo que cultivos agrícolas y/o producción animal, en alguna forma de arreglo espacial o secuencia temporal.
Sánchez (1998), señala que por agroforestería se entienden todos aquellos sistemas donde hay una combinación de especies arbóreas con especies arbustivas o herbáceas, generalmente cultivadas. Así, por ejemplo, el silvopastoreo es un tipo de agroforestería que implica la presencia de animales directamente pastando entre o bajo árboles.

Los sistemas agroforestales, tal y como lo expresa Iglesias (1999:287), pueden ser conceptualizados como una serie de sistemas y tecnologías del uso de la tierra, en las que se combinan árboles con cultivos agrícolas y/o pastos, en función del tiempo y el espacio, para incrementar y optimizar la producción en forma sostenida.

Existen diferentes puntos de vista para clasificar los sistemas agroforestales. La clasificación ha estado en función la estructura en el espacio, su diseño a través del tiempo, la importancia relativa y la función de los diferentes componentes, los objetivos de la producción y las características sociales y económicas prevalecientes (Iglesias, 1999:289).

Según Nair (1985:97), los sistemas agroforestales pueden ser clasificados en cuatro grupos, dependiendo de los aspectos estructurales y funcionales:

- Sistemas agrosilviculturales: combinaciones de árboles con cultivos. En estos se encuentran los cultivos en callejones, árboles de sombra sobre cultivos perennes, huertos caseros, barbechos mejorados, producción de leña, entre otros.

- Sistemas silvopastoriles: combinación de árboles con ganadería. Se diferencian las cercas vivas, pastos con 
árboles, banco de proteína, animales con producción de madera.

- Sistemas agrosilvopastoriles: utilización de árboles con cultivos y ganadería, como por ejemplo los huertos caseros con animales, hileras de arbustos para alimentación animal y protección del suelo, y producción integrada de cultivos.

- Otros: apicultura con árboles, lotes de árboles multipropósitos y acuacultura en mangles.

Sánchez (1998), clasifica los sistemas agroforestales de acuerdo al componente pecuario, de la siguiente manera:

- Pastoreo en bosques naturales.

- Pastoreo en plantaciones forestales para madera.

- Pastoreo en huertos.

- Pastoreo en plantaciones de árboles con fines industriales.

- Praderas con árboles o arbustos forrajeros en las praderas (cercas vivas, banco de proteína e inclusión de leguminosas forrajeras arbóreas directamente).

- Sistemas integrados mixtos con árboles forrajeros o multipropósito para corte.

- Sistemas agroforestales especializados para la producción animal bovina intensiva.

Cada uno de estos arreglos agroforestales genera beneficios particulares, los cuales deben ser tomados en consideración cuando se toma la decisión de adoptar esta tecnología. Sin embargo, lo más importante la decisión debe estar basada en las condiciones agroecológicas de la zona y a la capacidad de ofrecer el manejo más adecuado y a las especies asociadas, tanto animal como vegetal.

\section{El desarrollo sostenible como modelo de ética ambiental}

Considerar la Ética Ambiental en el ámbito de los sistemas agroforestales demanda una nueva manera de ver y entender el desarrollo sostenible y la conservación del ambiente. Tal intento no sería posible si no se construye junto a los productores las cosmovisiones de su permanencia en un ecosistema determinado.

En este sentido, Sepúlveda et al. (1998:10), plantean la perspectiva ecológica y ética como una relación entre sistemas ecológicos de gran cobertura y dinamismo, tendientes a fomentar los siguientes principios:

a. La vida humana debe continuar indefinidamente;

b. Las individualidades humanas deben crecer y multiplicarse;

c. Las particularidades culturales deben sobrevivir;

d. Las actividades humanas deben ser procesadas dentro de límites que no pongan en peligro la diversidad, complejidad y funciones del sistema ecológico que sirve de base a la vida humana.

En sus postulados, el desarrollo sostenible abarcar tres componentes básicos, los cuales han sido descritos por Reed (1996:40):

- Componente económico de la sostenibilidad: exige que las sociedades se encaminen por sendas de crecimiento económico que generen un verdadero aumento del ingreso, y no apliquen políticas a corto plazo que conduzcan al empobrecimiento a lar- 
go plazo. Requiere que las sociedades generen un flujo óptimo de ingresos a la vez que mantienen las exigencias básicas per cápita. La economía sostenible requiere de un enfoque diferenciado respecto al crecimiento y exige, además, internalizar todos los costos, incluyendo los sociales y ambientales relacionados con la producción y disposición de los bienes, implantando, el principio del costo total.

- Dimensión Social: presupone que la equidad y una comprensión de la interdependencia de las comunidades humanas, son requisitos básicos para una calidad de vida aceptable que, en último caso, es el principal objetivo del desarrollo. La equidad social significa asegurar que todas las personas tengan acceso a la educación y tengan la oportunidad de hacer contribuciones a la sociedad que sean productivas y justamente remuneradas. Esta dimensión exige la activa participación política de todos los sectores sociales y aprovechar el conocimiento y experiencias de las poblaciones, y por consiguiente, fortalecer la capacidad de los grupos sociales para moldear y manejar sus propias vidas.

- Dimensión Ambiental: se fundamenta en el mantenimiento de la integridad y, por lo tanto, de la productividad a largo plazo de los sistemas que mantienen la infraestructura ambiental y, por extensión, la vida en el planeta. Meyer y Meyer (1997:194), afirman que el desarrollo sostenible apunta a promover la eficacia económica y la equidad social, mientras que se asegure y refuerce la integridad ecológica y la identidad cultural.

\section{El hombre frente a la agricultura}

La necesidad cada vez creciente de suministrar alimentos para la humanidad, constituye una constante mundial. La estrategia para dar respuesta a esta situación, ha sido que el hombre ha desarrollado diferentes enfoques tecnológicos con el propósito de incrementar la oferta de productos agrícolas, tanto de origen animal como vegetal. Como consecuencia del uso indiscriminado de tecnologías, a mediados del siglo XX la producción agrícola experimentó un período de gran crecimiento, conocido como la "revolución verde".

Esta revolución consistía en que el hombre, frente a la agricultura, tendió a aumentar los rendimientos de los cultivos a través del uso de agroquímicos, maquinarias y mejoramiento genético de las especies vegetales. La explosión de estas prácticas trajo consigo el deterioro del ambiente, pérdida de la biodiversidad, degradación de suelos y contaminación de aguas subterráneas y superficiales. Se observa en este período que la actitud del hombre frente a su ambiente, podía considerarse como contraria a la conservación medioambiental.

Así, la ganadería en Latinoamérica, por ejemplo, se ha basado en la adaptación de modelos desarrollados en otras latitudes y los sistemas de producción bovina bajo pastoreo, han causado un gran daño al medio ambiente y a la biodiversidad. En consecuencia, tal y como lo plan- 
tea Howard - Borjas (1995:92), esta situación ha promovido la emigración de la población rural hacia las ciudades. La producción de carne y leche por unidad de área, entonces, ha llegado a un límite que parece insuperable con las estrategias de la revolución verde (Sánchez, 1998).

En respuesta a este hecho, el hombre actual y postmoderno, está intentando cambiar su conducta frente al ambiente, por lo que nace una corriente global sobre la protección del planeta y de sus recursos naturales. A este movimiento se le denominó la "revolución azul". En esta revolución se plantea la necesidad de trabajar hacia la sostenibilidad de los sistemas de producción, con la consecuente protección medioambiental y, de esta manera, utilizar los recursos presentes sin comprender la supervivencia de las futuras generaciones.

La ganadería por su parte, y en especial los sistemas de doble propósito, entendidos como sistemas de producción de leche y carne bovina, no han permanecido ajenos a esta nueva realidad y sobre todo, a lograr la sostenibilidad. Como parte de las estrategias implementadas en este sector agrícola, la adopción de sistemas agroforestales se perfila como una alternativa económicamente factible, socialmente justa y ambientalmente sana.

Hoy el hombre ha buscado el camino hacia los sistemas agroforestales, los cuales ofrecen una alternativa sostenible para aumentar la biodiversidad, y para aumentar los niveles de producción de manera significativa; ello con la intención de erradicar los sistemas de producción que ponían en peligro su permanencia en el tiempo, y por consiguiente, deteriora- ron el ambiente y sus ingresos económicos eran cada vez menores.

\section{Ecología y beneficios socioeconómicos: una relación posible}

Desde la perspectiva ecológica, plantea Wyant (1997:22), que la integración de los árboles en sistemas agrícolas en los sistemas agroforestales, puede ser vista como el pase hacia un ecosistema de creciente integridad ecológica, ya que se puede establecer un rango normal en la dinámica de los ecosistemas intervenidos.

Los beneficios humanos de los procesos ecológicos pueden ser utilizados aprovechando la diversidad de bienes y servicios generados por un ecosistema saludable, o permitiendo el desarrollo continuo de la integridad del ecosistema del bosque agroforestal.

Al respecto, menciona Leakey (1997:24), las diferentes prácticas agroforestales pueden ser vistas como fases en el desarrollo de un agroecosistema productivo, similar a la dinámica normal de los ecosistemas naturales. Con el tiempo, los sistemas agroforestales pueden considerarse como un bosque maduro, con creciente integridad ecológica.

Los sistemas agroforestales deben ser considerados como un puente entre la conservación y el desarrollo. Al respecto, señalan Arce y García (1997:11), que entender la función de los elementos y de los procesos entre ecosistemas naturales y los agroecosistemas, así como sus interacciones, debería llevar, en teoría, a una mejor comprensión de la necesidad de preservar los recursos silvestres. 
Current (1997:12), plantea que resulta muy difícil poder cuantificar los beneficios ambientales y sociales de los sistemas agroforestales, la poca superficie aislada que es dedicada a éstos en las fincas, y no medir la conservación del suelo y de otros recursos ambientales. En algunos países latinoamericanos, estos sistemas proveen diferentes productos arbóreos, protegiendo al bosque natural, como por ejemplo, en El Salvador, una comunidad que producía carbón, ahora está utilizando los árboles de los sistemas agroforestales.

El desarrollo de los sistemas agroforestales supone la utilización de árboles y arbustos que, en su mayoría, son especies fijadoras de nitrógeno atmosféri$\mathrm{co}$, los cuales se asocian con otros cultivos y especies animales como el ganado bovino u ovino.

Botero y Russo (1998), señalan que los sistemas agroforestales que han incorporado especies fijadoras de nitrógeno como las leguminosas, se crean interacciones biológicas, ecológicas y económicas que mejoran la productividad de los sistemas de producción.

Otras ventajas de los sistemas agroforestales con componente pecuario, dirigidos a intensificar la producción animal, son resumidas por Sánchez (1998), de la siguiente manera: a) mayor cantidad y calidad del alimento, b) microclima favorable, c) expansión de la biodiversidad, d) potencial de fijación de nutrientes, entre otros.

Desde el punto de vista económico, los sistemas agroforestales producen beneficios importantes. Hernández et al. (1998), evaluaron el efecto de la inclusión, en banco de proteína de leucaena (leguminosa forrajera arbórea), sobre el mejoramiento de gramíneas naturales y el aumento de peso en animales de ceba, obteniendo ganancias de peso de $715 \mathrm{~g} / \mathrm{animal} / \mathrm{día}$ y un incremento en la producción de carne por hectárea del $51 \%$.

Nelson (1998:251), a través de modelos de simulación, obtuvo valores de VAN positivos a una tasa de actualización del $25 \%$, en sistemas agroforestales en Filipinas, considerando el cultivo de maíz y de Gliricidia sepium.

Asimismo, Lamela et al. (1999: 339), estudiaron la producción de leche en un sistema agroforestal, encontrando que el empleo del banco de proteína permite obtener producciones de leche de 9 I/vaca/día, con un aceptable comportamiento reproductivo de los animales.

Como puede observarse, los resultados derivados de la utilización de sistemas agroforestales, arrojan beneficios ambientales importantes, sobre todo los relacionados con la fijación de nitrógeno en el suelo y la conservación de los recursos naturales. En el plano económico, estos sistemas ayudan a mejorar la productividad de los sistemas de producción.

De igual manera, los sistemas agroforestales generan bienes que no tienen valor de mercado, así se obtiene por ejemplo, un beneficio por la no utilización de recursos naturales, es decir, no se comprometen, conservándolos para las generaciones futuras. También, estos beneficios pueden centrarse en la disposición a pagar por mantener la posibilidad de poder disfrutar un determinado bien ambiental en el futuro, aunque no se disfrute actualmente. 


\section{Consideraciones finales}

La incorporación del tema ético en las organizaciones resulta trascendental en los nuevos tiempos de las sociedades humanas. Los enfoques sobre el desarrollo organizacional moderno apuntan hacia la armonía interna de los miembros que laboran en éstas, pero a su vez, debe respetarse la relación empresa-hombre-sociedad.

Anteriormente se ha señalado las bondades de la incorporación de la agroforestería en los sistemas de producción y se ha abordado el tema de la ética ambiental como cuestión fundamental. Sin embargo, la adopción de esta tecnología resulta incipiente o nula en los sistemas de producción, bien porque se desconoce su existencia y utilización o simplemente porque los gerentes no están comprometidos con el cambio paradigmático que ocurre en el mundo globalizado.

No obstante, ese constante cuestionamiento sobre lo qué se debe hacer o no se debe hacer, ese conflicto entre lo que es y lo que debe ser, ha llamado la atención de la gerencia moderna en los sistemas de producción y de personas preocupadas por la conservación medioambiental. Así, la agricultura también ha llegado a la postmodernidad o el "shock" del futuro como la define Pulido (1998:23), la cual se concibe como el flujo de situaciones donde priva el concepto de transitoriedad y es la adaptación al cambio y no lo permanente, lo que genera modelos de referencia.

La gerencia de los sistemas de producción es llamada a considerar los sistemas agroforestales como un manejo que el hombre postmoderno otorgue a la actividad agrícola, por lo que se hace nece- sario, ubicar la visión conservacionista de la agroforestería, bajo un paradigma fluctuante, donde los gerentes o líderes tienen la misión de transmitir principios, criterios y valores.

Los gerentes de sistemas de producción apoyados en la ecología, la ética ambiental y la agroforestería, han de encarar los vaivenes de esta época, para crear constancias dentro de la inconstancia de referentes permanentes.

Es por esto, que los promotores de la agroforestería o gerentes de sistemas de producción que bien pudiesen ser clasificados como líderes transformacionales, les toca un momento ético, cultural y filosófico difícil, toda vez que se ha de lograr en una época de constantes cambios la formación y perpetuación de hombres comprometidos con la agroforestería.

En la postmodernidad, tal y como lo propone Leakey (1997:24), los gerentes de los sistemas de producción enfrentan un nuevo reto: la integración de algunas de las prácticas agroforestales actuales en sistemas de uso de la tierra productivos y sostenibles, como alternativa tecnológica y ética para aliviar la pobreza. Por lo tanto, son los gerentes de los sistemas de producción los llamados a promover cambios en el hacer, pensar y sentir de los productores, para que puedan enfrentar con éxito el difícil reto de producir más con menos recursos, en un mundo postmoderno y globalizado.

Finalmente, el gerente de sistemas de producción debe hacer de la ética ambiental parte de su filosofía de su vida e incorporar en sus valores, la protección del medio ambiente, para estar en armonía con la sociedad en la cual se desarrolla su organización. 


\section{Bibliografía citada}

Arce, R., y A. García (1997). La agroforestería social: un nexo entre la conservación y el desarrollo sostenible. Agroforestería en Las Américas. Vol. 4, No. 16, Costa Rica, pp. 15-21.

Botero, Raúl y Ricardo Russo (1998). Utilización de árboles y arbustos fijadores de nitrógeno en sistemas sostenibles de producción animal en suelos ácidos tropicales. En: Conferencia Electrónica de la FAO sobre Agroforestería para la producción animal en Latinoamérica. www.fao.org. 08/03/01.

Burley, J., y A.W. Speedy (1998). Investigación Agroforestal - Perspectivas Globales. En: Conferencia Electrónica de la FAO sobre Agroforestería para la producción animal en Latinoamérica. www.fao.org. 08/03/01.

Cañas - Quiroz, R. (1998). Ética general y Ética profesional. En: Conferencia Electrónica de Acta Académica. www.uaca.ac.cr/acta/1998nov/rcanas.htm. 20/04/01.

Current, D. (1997). ¿Los sistemas agroforestales generan beneficios para las comunidades rurales?. Resultados de una investigación en América Central y el Caribe. Agroforestería en Las Américas. Vol. 4, No. 16, Costa Rica, pp. 8-14.

Degioanni, A. (2001). Ambiente, región y deber. www.unrc.edu.ar/publicar/19/dosier8.htm. 20/04/01.

Díaz, J. (2000). La ética en la gerencia, vista a través del desarrollo organizacional.

Revista Venezolana de Gerencia, Año 5, No. 10, Venezuela, La Universidad del Zulia, Vice Rectorado Académico, pp. 49-91.

Etkin, J. (1993). La doble moral de las organizaciones. Los sistemas perver- sos y la corrupción institucionalizada. Madrid: Mc Graw Hill. 328 pp.

Ferrete, Carmen (1996). Economía y ética: La problematicidad del Desarrollo Sostenible. www.uv.es/adrfp/dilema/dilema5c.htm 20/04/01.

Hernández, I, Milagros Milera, L. Simón, D. Hernández, J. Iglesias, L. Lamela, Odalys Toral, C. Matías y Geraldine Francisco (1998). Avances en las investigaciones en sistemas silvopastoriles en Cuba. En: Conferencia Electrónica de la FAO sobre Agroforestería para la producción animal en Latinoamérica. www.fao.org. 08/03/01.

Howard - Borjas, P. (1995). Cattle and crisis: the genesis of unsustainable development in Central America. En: Reforma Agraria, colonización y cooperativas. FAO, Roma, pp. 89-116.

Iglesias, J. (1999). Sistemas de Producción Agroforestales. Conceptos generales y definiciones. Pastos y Forrajes. No. 22, Cuba, Estación Experimental de Pastos y Forrajes "Indio Hatuey", pp. 287-305.

Lamela, C., C. Matías y A. Gómez (1999). Producción de leche en un sistema con banco de proteína. Pastos y Forrajes. No. 22. Cuba, Estación Experimental de Pastos y Forrajes "Indio Hatuey", pp. 339-345.

Leakey, R. (1997). Reconsiderando la definición de agroforestería. Agroforestería en Las Américas. Vol. 4, No. 16. Costa Rica, pp. 23-24.

Meyer H; Von Meyer (1997). Integrated rural development dimensions of a new approach. Zeitschrift fur Kulturtechnik und Landentw. Vol. 38, No. 5. Alemania, pp. 1993 - 197.

Nair, P. (1985). Classification of agroforestry systems. Agroforestry Systems. No. 3, Holanda, p. 97. 
Nelson, R. (1998). Cost - benefit analysis of atternative forms of hedgerow intercropping in the Philipine uplands. Agroforestry Systems. No. 39, Holanda, pp. 241-262.

Pulido, H. (1998). La Ética y los Valores a la luz de la postmodernidad. Docencia, Investigación, Extensión. Vol. 2, № 1, Venezuela, Universidad Nacional Experimental Libertador, pp. 21-26.

Reed, D. (1996). Ajuste Estructural; Ambiente y Desarrollo Sostenible. Caracas. Editorial CENDES, pp. 56-63.

Sánchez, M. (1998). Sistemas Agroforestales para intensificar de manera sostenible la producción animal en Latinoaméri- ca tropical. En: Conferencia Electrónica de la FAO sobre Agroforestería para la producción animal en Latinoamérica. www.fao.org. 08/03/01.

Sepúlveda S.; Castro, A. y Rojas, P. (1998). Estimación del Nivel de Desarrollo Sostenible: El caso de Costa Rica.. Cuadernos Técnicos 5. Costa Rica, IICA, pp. 96.

Vidal, M; P. Santidrian (1990). Ética Personal. Las Actitudes éticas. Caracas, Paulinas, pp. 121.

Wyant, J. (1997). Agroforestería: una perspectiva ecológica. Agroforestería en Las Américas. Vol. 4, No. 16. Costa Rica, pp. 22-23. 